\title{
5-azacytidine induces cardiac differentiation of P19 embryonic stem cells
}

\author{
Seung-Cheol Choi ${ }^{1}$, Jihyun Yoon ${ }^{1}$, \\ Wan-Joo Shim ${ }^{1}$, Young-Moo Ro ${ }^{1}$ \\ and Do-Sun Lim $^{1,2}$ \\ 'Department of Cardiology \\ Korea University Medical College \\ Seoul 136-705, Korea \\ ${ }^{2}$ Corresponding author: Tel, 82-2-920-5445; \\ Fax, 82-2-927-1478; E-mail, dsImd@kumc.or.kr
}

Accepted 1 November 2004

Abbreviations: 5-aza, 5-azacytidine; EB, embryoid body; EC, embryonal carcinoma; ES, embryonic stem

\section{Abstract}

The P19 embryonal carcinoma cell line is a useful model cells for studies on cardiac differentiation. However, its low efficacy of differentiation hampers its usefulness. We investigated the effect of 5-azacytidine (5-aza) on P19 cells to differentiate into a high-efficacy cardiomyocytes. Embryoid-body-like structures were formed after 6 days with $1 \mu \mathrm{M}$ of 5 -aza in a P19 cell monolayer culture, beating cell clusters first observed on day 12, and, the production of beating cell clusters increased by $80.1 \% \quad(29$ of 36-wells) after 18 days. In comparison, the spontaneous beating cells was $33.3 \%$ (12 of 36 -wells) for the untreated control cells. In response to $1 \mu \mathrm{M}$ of 5-aza, P19 cells expressed bone morphogenetic protein-2 (BMP-2), BMP-4, Bmpr1a and Smad1 at day 6 or 9 , and also cardiac markers such as GATA-4, Nkx2.5, cardiac troponin $I$, and desmin were up-regulated in a time-dependent manner after induction of BMP signaling molecules. Immunocytochemistry revealed the expression of smooth muscle $\alpha$-actin, sarcomeric $\alpha$-actinin, cardiac myosin heavy chain, cardiac troponin $\mathrm{T}$ and desmin, respectively. The proportion of sarcomeric $\alpha$-actinin positive cells accounted for $6.48 \%$ on day 15 after 5 -aza exposure as measured by flow cytometry. This study has demonstrated that 5-aza induces differentiation of $\mathrm{P} 19$ cells into cardiomyocytes in a confluent monolayer culture in the absence of prior embryoid formation and dimethyl sulfoxide exposure, depending in part on alteration of BMP signaling molecules. These results suggest that 5-aza treatment could be used as a new method for cardiac differentiation in $\mathrm{P} 19$ cells.

Keywords: aza compounds; bone morphogenetic proteins; cardiac myocytes, cell differentiation; gene expression; stem cells

\section{Introduction}

The pluripotent P19 embryonal carcinoma (EC) cell line is derived from a teratocarcinoma formed in $\mathrm{C} 3 \mathrm{H} / \mathrm{He}$ mice, and this cell line has the ability to differentiate into all three germ layers (Martin, 1975; McBurney, 1993). P19 cells have been used as a model system for studying cardiac differentiation because these cells can differentiate into cardiomyocytes in vitro. They provide some advantages over embryonic stem (ES) cells due to the ease of the cell culture system, which can sustain an undifferentiated cell state without a feeder-cell layer. In addition, it has the ability to facilitate cell differentiation in large quantities and the cell line has a susceptibility to incorporate and express ectopic genes (Van der Heyden and Defize, 2003). The combination of embryoid body (EB) formation and dimethyl sulfoxide (DMSO) has been mainly used to induce the cardiac differentiation of P19 cells (McBurney et al., 1982). In addition to DMSO, other factors such as retinoic acid, butyrate, 6-thioguanine and co-culture of P19 cells with the P19 derived visceral endoderm-like cell line (END-2) have been found to induce the cardiac differentiation of P19 cells (Edwards and McBurney, 1983; Mummery et al., 1991; Rodriguez et al., 1994). Paquin et al. (2002) have recently reported that oxytocin induced cardiomyocyte differentiation in P19 cells via the DMSO action pathway. However, the low efficiency of P19 cells to differentiate is a major limitation.

5-azacytidine (5-aza), a cytosine analog, was originally developed and tested as a nucleoside antimetabolite for acute myelogenous leukemia (Sorm et al., 1964; Cihak, 1974). This compound is an effective DNA hypomethylating agent, and it is capable of altering the expressions of certain genes (Mohandas et al., 1981; Branch et al., 1996) or regulating cell 
differentiation (Jones and Taylor, 1980; Bartolucci et al., 1989). Several studies have recently found that mesenchymal stem cells can be transformed into cardiomyocytes after an exposure to 5-aza (Makino et al., 1999; Hakuno et al., 2002; Rangappa et al., 2003). In addition, human ES cells (Xu et al., 2002) and stem cell antigen-1 (Sca-1) ${ }^{+}$cardiac progenitor cells (Oh et al., 2003) were differentiated into cardiomyocytes in response to 5-aza or 5-aza-2'-deoxycytidine treatment.

On the basis of the previous studies, we hypothesized that demethylation itself is possibly one of the important factors that could induce or enhance cardiac differentiation by regulating the expressions of silent genes. In the present study, we have demonstrated that 5-aza induces cardiac differentiation of P19 cells in a confluent monolayer culture even in the absence of prior EB formation and DMSO exposure; this was confirmed by the up-regulation of BMP signaling molecules and cardiac marker genes, and the increase of beating cell clusters after 5-aza exposure.

\section{Materials and Methods}

\section{Culture and cardiac differentiation of P19 cells}

The P19 cells were obtained from the American Type Culture Collection (ATCC; Rockville, MD) and cultured in $\alpha$-minimal essential medium (MEM) (Invitrogen, Grand Island, NY) supplemented with $10 \%$ fetal bovine serum (FBS; Invitrogen), $100 \mathrm{U} / \mathrm{ml}$ penicillin (Invitrogen) and $100 \mu \mathrm{g} / \mathrm{ml}$ streptomycin (Invitrogen). All the cells were passaged at 2- to 3-day intervals. To induce differentiation, the cells were cultured in medium containing different concentrations $(0.5,1,2$, 5 and $10 \mu \mathrm{M}$ ) of 5 -aza (Sigma, St. Louis, MO) at a density of $1.0 \times 10^{3}$ cells in each well of 12-well dishes. The medium containing 5-aza was replenished at 2-day intervals. After 5-aza treatment, the morphological changes of P19 cells were examined under a Nikon inverted microscope (Nikon, Tokyo, Japan) that was equipped with phase-contrast objectives and a digital camera (Nikon). To examine cell viability after 5 -aza treatment, $1.0 \times 10^{3}$ cells/well were seeded in 12-well dishes and exposed for 4,7 and 10 days to $0.5,1$ and $2 \mu \mathrm{M}$ of 5 -aza. The cells were detached with $0.25 \%$ trypsin, and then stained with $0.2 \%$ trypan blue (Invitrogen). The cells were then counted by using a hemocytometer.

Differentiation was also induced with $1 \%$ DMSO. Briefly, the cultures were initiated by plating cells into $10-\mathrm{cm}$ bacteriological grade Petri dishes at $1 \times 10^{6}$ cells in $14 \mathrm{ml}$ of medium that was supplemented with $1.0 \%$ DMSO (Sigma) from day 0 to day 4. At day 4 , cell aggregates were transferred to $6-\mathrm{cm}$ tissue culture dishes and allowed to adhere. The culture medium was refreshed every second day.

\section{Reverse transcription-polymerase chain reaction (RT-PCR)}

The total cellular RNA was extracted from the cells with Trizol (Gibco-BRL, Grand Island, NY). Contaminating DNA was digested by $1 \mathrm{U}$ of RQ1 DNase (Promega, Madison, WI) as recommended by the manufacturer. The first-strand cDNA was synthesized from $0.5 \mu \mathrm{g}$ of DNase-treated total RNA using $0.5 \mu \mathrm{g}$ random hexamers (Invitrogen), and $200 \mathrm{U}$ moloney murine leukemia virus (M-MLV) reverse transcriptase (Invitrogen) at $37^{\circ} \mathrm{C}$ for $60 \mathrm{~min}$ in a volume of $20 \mu \mathrm{l}$. The first strand CDNA $(1 \mu \mathrm{l})$ was used for PCR amplification in a $25 \mu$ reaction mixture. PCR was performed under the following conditions: $94^{\circ} \mathrm{C}$ for 30 $\mathrm{s}, 60^{\circ} \mathrm{C}$ for $40 \mathrm{~s}$, and $72^{\circ} \mathrm{C}$ for $40 \mathrm{~s}$, with an additional 7 -min incubation at $72^{\circ} \mathrm{C}$ after cycle completion. The PCR reaction was carried out for 27 cycles with glyceraldehyde-3-phosphate dehydrogenase (GAPDH), for 30 cycles with GATA-4, octamer-binding protein 4 (Oct-4) and Nanog, and for 33 cycles with bone morphogenetic protein-2 (BMP-2), BMP-4, type $1 \mathrm{~A}$ receptor for BMP (Bmpr1a), cardiac troponin I, desmin, $\beta$-myosin heavy chain $(\beta-M H C), N k x 2.5$, Smad1 and Smad5 primers. The primers used for PCR were as follows: BMP-2, 5'-CTCCACAAACGAGAAAAGCG-3' and 5'-CATGCCTTAGGGATTTTGGA-3'; BMP4, 5'-TGATACCTGAGACCGGGAAG-3' and 5'-ACTCCTCACAGTGTTGGCTC-3'; Bmpr1a, 5'-CGCTATGGAGAAGTATGGATGG-3' and 5'-GGTCAGCAATACAGCAACTTCC-3'; cardiac troponin I, 5'-GAAGCAGGAGATGGAACGAG-3' and 5'-TTAAACTTGCCACGGAGGTC-3'; desmin, 5'-ATGAGCCAGGCCTACTC GTC-3' and 5'-GCGCACCTTCTCGATCTAGT-3'; GATA-4, 5'-CTGTCATCTCACTATGGGCA-3' and 5'-CCAAGTCCGAGCAGGAATTT-3'; Oct-4, 5'-GGCGTTCTCTTTGGAAAGGTTTC-3' and 5'-CTCGAACCACATCCTTC TCT-3'; $\beta$-MHC, 5'-ACCCCTACGATTATGCG-3' and 5'-GTGACGTACTCGTTGCC-3'; Nanog, 5'-CACCCAC CCATGCTAGTCTT-3' and 5'-ACCCTCAAACTCCTGG TCCT-3'; Nkx2.5, 5'-AGCAACTTCGTGAACTTTG-3' and 5'-CCGGTCCTAGTGTGGA-3'; Smad1, 5'-AGCC CAACAGCCACCCGT-3' and 5'-GCAACTGCCTGAAC ATCTCCT-3'; Smad5, 5'-GCTGAACCCCATTTCTTCT G-3' and 5'-CGTTCCAGGTTAAGATCAATGC-3'; GAPDH, 5'-TTCACCACCATGGAGAAGGC-3' and 5'-GGC ATGGACTGTGGTCATGA-3'.

\section{Immunocytochemistry}

For the immunostaining procedure, the cells were grown on glass coverslips coated with $0.1 \%$ gelatin in 12-well dishes. They were then fixed by a $20-\mathrm{min}$ incubation in PBS containing $4 \%$ paraformaldehyde 
(Sigma), and they were next rinsed in PBS containing $0.1 \%$ Tween 20 (PBT) 3 times. The fixed cells were permeabilized for $30 \mathrm{~min}$ in PBS containing $0.5 \%$ Triton X-100, and they were blocked with $10 \%$ normal goat serum (Gibco-BRL) in PBT for $1 \mathrm{~h}$. The cells were then incubated with smooth muscle $\alpha$-actin (Sigma), sarcomeric $\alpha$-actinin (Sigma), cardiac myosin heavy chain (Biogenesis, Kingston, NH), cardiac troponin $\mathrm{T}$ (Sigma) and desmin (Sigma) at $4{ }^{\circ} \mathrm{C}$ overnight in $2 \%$ normal goat serum in $\mathrm{PBT}$, respectively. After a washing in PBT, the cells were incubated with fluorescin isothiocyanate (FITC) conjugated rabbit-anti mouse IgG (Sigma) as the secondary antibody for 1 $\mathrm{h}$ at room temperature. The cells were covered with Vectashield (Vector, Burlingame, CA) containing 1.5 $\mu \mathrm{g} / \mathrm{ml}$ of 4', 6-diamidino-2-phenylindole (DAPI), and the immunofluorescence was visualized using a Zeiss Axioscope microscope (Zeiss, Oberkochen, Germany).

\section{Flow cytometry}

The P19 cells were treated with $1 \mu \mathrm{M}$ of 5 -aza for 15 days. The media was removed from the flasks, and the cell layers were washed twice with PBS and detached from the flasks by incubating them with a solution of $0.25 \%$ trypsin-EDTA for $5 \mathrm{~min}$ at room temperature. The cells were recovered by centrifugation, then permeabilized in cold methanol for 15 min on ice, fixed in $2 \%$ paraformaldehyde for $20 \mathrm{~min}$ on ice and washed twice with PBS containing $0.2 \%$ Tween 20. Cells were next incubated with sarcomeric $\alpha$-actinin antibody and also with FITC-conjugated rabbit-anti mouse $\lg$. All incubations with antibodies were performed for $1 \mathrm{~h}$, after which time the cells were washed with PBS containing $0.2 \%$ Tween 20 . Ten thousand cells were analyzed on a Becton Dickinson Vantage instrument (San Jose, CA) using Cell-Quest software.

\section{Statistical analysis}

Values are reported as means \pm SE. Statistical significance was evaluated with an unpaired Student's $t$-test for comparison between 2 groups or with ANOVA for comparison and contrast between multiple groups. Significance level was set at a $P$ value $<$ 0.05 .

\section{Results}

\section{Induction of cardiac differentiation in the P19 confluent monolayer culture by 5 -aza}

The mesenchymal stem cells were differentiated into cardiomyocytes by 5 -aza, and this may have occurred by the demethylation of the DNA (Makino et al.,
1999). We investigated the effects of 5-aza on cardiac differentiation of the P19 cells under different conditions. First, P19 cell aggregates were formed in bacteriological dishes from day 0 to day 4 in the presence of $1.0 \% \mathrm{DMSO}$, and the cells were further treated with $10 \mu \mathrm{M}$ of 5 -aza at the differentiation day 1 to $3(4+1-3$, where 4 represents a four day period prior to the treatment with DMSO), and 3 to $5(4+$ $3-5)$, or 5 to $7(4+5-7)$ in the cell culture dishes. The cells were harvested at differentiation day 12 (4 + 12) and then analyzed by RT-PCR with for the presence of cardiac markers such as GATA-4 and $\beta$-MHC. Treatment of P19 cells with $10 \mu \mathrm{M}$ of 5 -aza after the cardiomyogenic induction by $1.0 \%$ DMSO revealed a similar cardiac marker expression as those cells of the DMSO only treated control group (Figure 1A). Second, the P19 cells were exposed to different concentrations of 5-aza for consecutive 12 days after their aggregate formation in bacteriological dishes for 4 days in the presence of $1.0 \%$ DMSO. The expression of cardiac markers was very similar irrespective of the 5-aza concentrations, and it was similar to that of the DMSO only treated control cells (Figure 1B). Third, the P19 cells were exposed to 1, 5 and $10 \mu \mathrm{M}$ of 5 -aza for 15 consecutive days in the cell culture dishes to determine whether 5-aza can directly induce the cardiomyogenic differentiation without EB formation in the absence of $1.0 \%$ DMSO in the bacteriological dishes.

A significant induction of cardiac specific markers, such as GATA-4 and $\beta$-MHC, was detected when the P19 cells were exposed to $1 \mu \mathrm{M}$ of 5 -aza compared with results of the undifferentiated control, as noted by RT-PCR (Figure 1C). This result demonstrates that 5 -aza can induce cardiomyocyte differentiation in the P19 confluent monolayer culture which has high local cell densities in the absence of prior cellular aggregation and DMSO exposure. On the other hand, cells treated with 5 and $10 \mu \mathrm{M}$ of 5 -aza in the monolayer culture became necrotic (data not shown), whereas no growth inhibition was observed in the presence of $10 \mu \mathrm{M}$ of 5 -aza in the P19 cellular aggregates induced by the combination of prior $E B$ formation and DMSO (Figures $1 \mathrm{~A}$ and $1 \mathrm{~B}$ ).

To evaluate the effect of 5-aza on the growth of P19 cells, the cells seeded at an initial density of 1.0 $\times 10^{3}$ cells in 12 -well dishes were treated with 0.5 , 1 and $2 \mu \mathrm{M}$ of 5 -aza for 4,7 and 10 days. 5-aza treatment on the P19 cells revealed dose-dependent effects on the proliferation of P19 cells. No significant difference for the growth of P19 cells was observed at a concentration of $0.5 \mu \mathrm{M}$ of 5 -aza, although the average number of cells was decreased as compared with the 5-aza-untreated controls (Figure 2). 5-aza at a concentration of $1 \mu \mathrm{M}$ significantly inhibited the proliferation of P19 cells as compared with the 


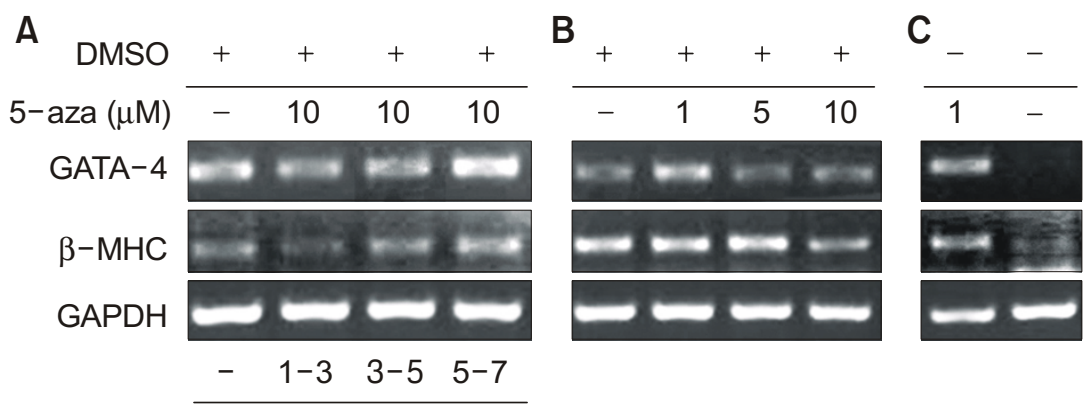

Days of 5-aza treatment

Figure 1. Effect of 5-aza treatment on cardiac differentiation of P19 cells. A. P19 cells were cultivated as aggregates from day 0 to day 4 in the presence of 1\% DMSO in bacteriological dishes. At day 4 , the cell aggregates were transferred to cell culture dishes and treated with $10 \mu \mathrm{M}$ of 5 -aza at differentiation day 1 to $3(4+1-3), 3$ to $5(4+3-5)$ or 5 to $7(4+5-7)$. The cells were harvested at differentiation day $12(4+12)$. B. P19 cells were cultivated as aggregates from day 0 to day 4 in the presence of $1 \%$ DMSO in bacteriological dishes. At day 4 , the cell aggregates were transferred to cell culture dishes and then treated for consecutive 12 days with 1,5 or $10 \mu \mathrm{M}$ of 5 -aza, respectively. The cells were harvested at differentiation day $12(4+12)$. C. P19 cells were directly exposed to $1 \mu \mathrm{M}$ of 5 -aza in the cell culture dishes without cellular aggregation and DMSO treatment, and then the cellular aggregrates were harvested at day 15. RT-PCR was performed with primers for GATA-4 and $\beta$-myosin heavy chain $(\beta-M H C)$ as cardiac specific markers, and GAPDH was used as the input control.

controls at day $4 \quad(70,043 \pm 4,473$ vs. $56,443 \pm$ $10,237$, respectively, $P<0.05$; Figure 2$)$, at day 7 $(1,866,000 \pm 139,929$ vs. $1,570,000 \pm 64,031$, respectively, $P<0.01$; Figure 2) and at day 10 $(3,980,000 \pm 235,797$ vs. $3,408,000 \pm 376,723$, respectively, $P<0.05$; Figure 2 ). The proliferation of P19 cells was more inhibited in the presence of 2 $\mu \mathrm{M}$ of 5 -aza as compared with the controls at day $4(70,043 \pm 4,473$ vs. $50,933 \pm 5,330$, respectively, $P<0.01$; Figure 2), at day $7(1,866,000 \pm 139,929$ vs. $1,284,000 \pm 143,108$, respectively, $P<0.001$; Figure 2) and at day $10 \quad(3,980,000 \pm 235,797$ vs. $3,304,000$ vs. 294,414 , respectively, $P<0.01$; Figure 2 ).

\section{Generation of beating cell regions from P19 cells induced by 5-aza}

Cellular aggregation is known to be a requirement for the DMSO-induced differentiation of P19 EC cells into cardiac myocytes (McBurney et al., 1982), and this process is necessary for initiating BMP signaling cascade that subsequently regulates $\mathrm{Nkx} 2.5$ activity (Jamali et al., 2001). To investigate how P19 cells can be differentiated into cardiac cells by 5 -aza treatment with the absence of prior EB formation and without exposure to DMSO, we examined the morphological changes of the P19 cells in the presence of $1 \mu \mathrm{M}$ of 5 -aza. P19 cells were seeded at a low density of $1.0 \times 10^{3}$ /well onto 12 -well dishes to allow those cells to differentiate into cardiac cells by delaying the cells proliferate to confluent state. After 6 days, the cells with differentiated phenotypes were observed (Figure 3A). After 9 days, EB-like structures with high cell densities packed in multiple layers were formed (Figure 3A). After 12 days, beating cell clusters were observed for the first time (Figure $3 A$ ). However, a similar morphological changes and beating cell clusters were occasionally observed on the indicated differentiation days in the control cells indicating that under this cultural conditions, the cells can spontaneously differentiate into cardiomyocytes in the absence of 5-aza treatment (data not shown). Further examination on the effect of 5-aza to enhance the production of beating cell clusters showed that the cumulative percentages of the wells containing beating cell clusters after plating with 5-aza-treated P19 cells were significantly higher than those of the controls at day $12(27.8 \pm 4.8$ vs. $5.6 \pm 4.8$, respectively, $P<0.01$; Figure $3 \mathrm{~B})$, at day $14(44.5 \pm 4.8$ vs. $16.7 \pm 8.3$, respectively, $P<0.05$; Figure $3 \mathrm{~B}$ ), at day $16(66.7 \pm 8.4$ vs. $30.5 \pm 3.9$, respectively, $P<0.01$; Figure $3 \mathrm{~B})$ and at day 18 (80.3 \pm 4.6 vs. $33.3 \pm 6.8$, respectively, $P<0.01$; Figure $3 \mathrm{~B})$. These results clearly demonstrate that formation of spontaneous beating cell clusters in the P19 confluent monolayer culture is enhanced in response to 5 -aza, suggesting that the alteration of gene expression by the demethylation process may be one of important factors for cardiac differentiation in embryonic P19 stem cells as well as in mesenchymal stem cells. 

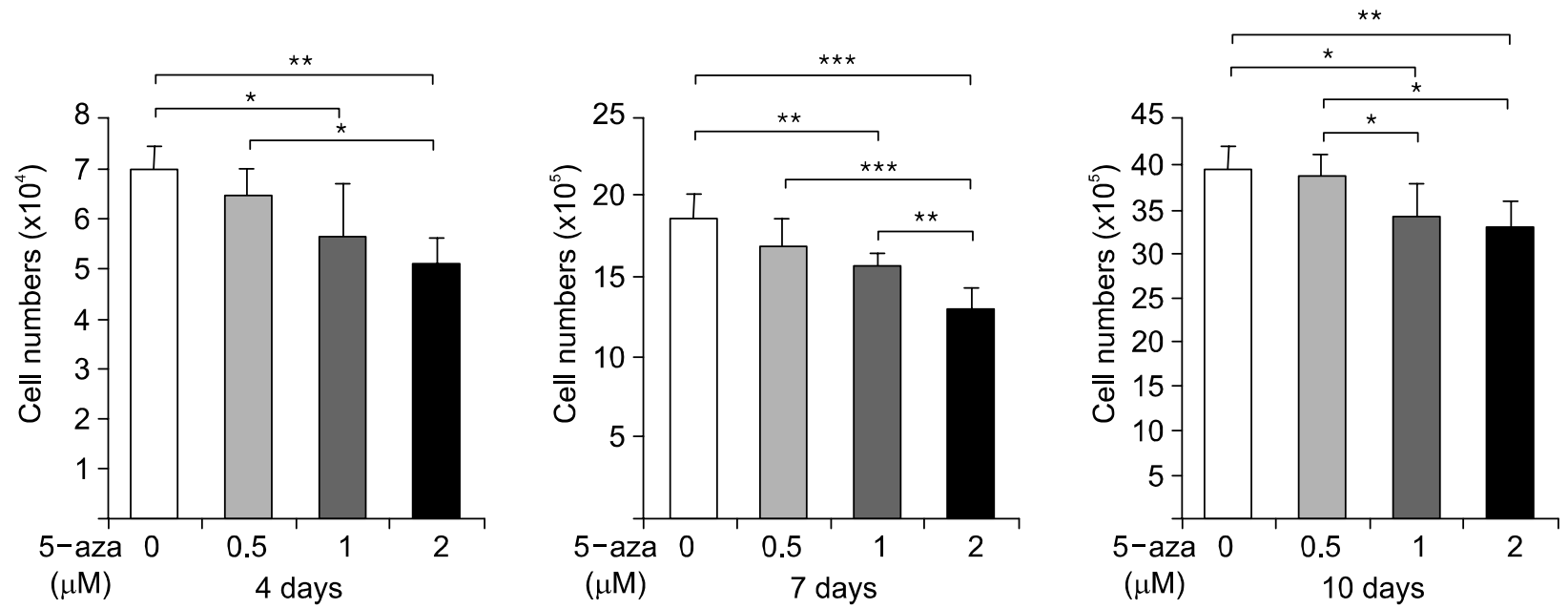

Figure 2. 5-aza inhibits P19 cell proliferation in dose-dependent manner. For cell growth measurement, $1.0 \times 10^{5}$ cells were plated in triplicate onto 12-well plates and exposed to $0.5,1$ and $2 \mu \mathrm{M}$ of 5-aza for 4,7 and 10 days. The cells were then trypsinized on the indicated day and counted by using a hemocytometer after staining with $0.2 \%$ trypan blue. ${ }^{*} P<0.05,{ }^{* *} P<0.01,{ }^{* * *} P<0.001$.
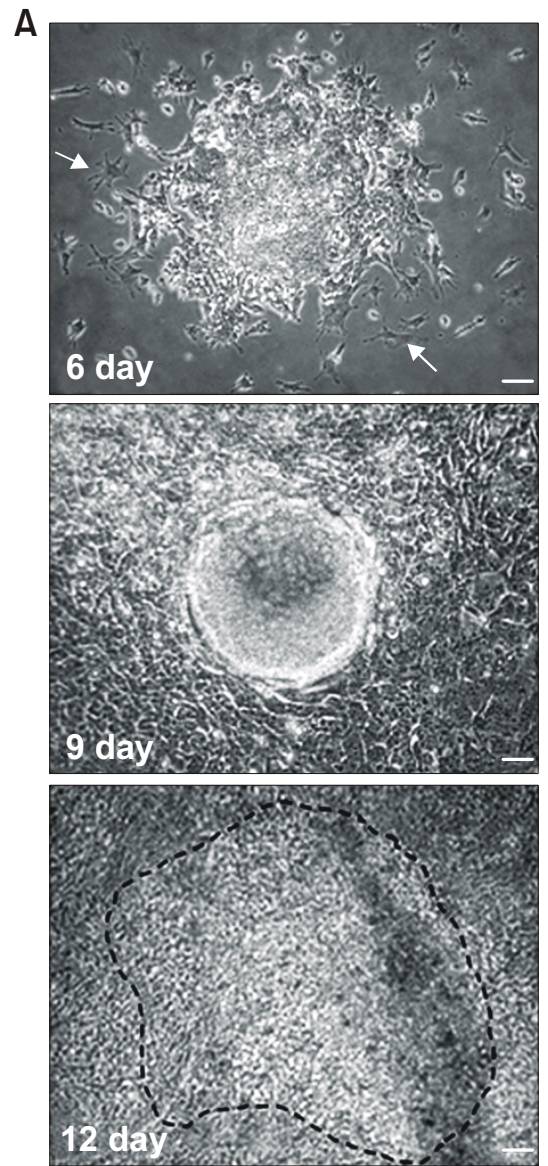

B

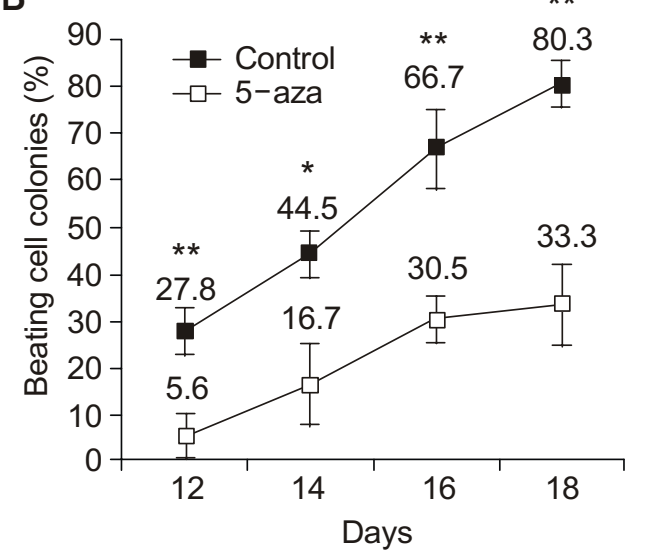

Figure 3. Examination of morphological changes of P19 cells, and the generation of beating cell colonies from P19 cells after 5 -aza treatment. P19 cells were seeded at a density of $1.0 \times 10^{3}$ cells/well onto 12-well plates and cultured for 18 days in the presence of $1 \mu \mathrm{M}$ of 5-aza. A. After 6 days, differentiated cell types (arrows) were found. After 9 days, embryoid body-like structures were formed. After 12 days, beating cell colonies were found, and they were marked with a dotted-line after being videotaped with a digital camera. Scale bar, $50 \mu \mathrm{m}$. B. Time course of the appearance of beating cell colonies in the 5-aza-treated P19 cells. The number of wells containing rhythmically contracting regions was scored from 12 to 18 day at 2-day intervals. The beating cell colonies from a total of 36 -wells were counted. The results are expressed as the mean percents \pm standard deviations. ${ }^{*} P<$ $0.05,{ }^{* *} P<0.01$ as compared with corresponding 5 -aza-untreated controls.
Alteration of BMP signaling and expression of cardiac specific genes in 5-aza-treated P19 cells To investigate the effect of 5 -aza on cardiomyogenic differentiation of the P19 cells, the expressions of cardiac marker genes were examined by using RT-PCR. The results of RT-PCR revealed that the 


\begin{tabular}{|c|c|c|}
\hline Control & 5-aza & \\
\hline $\begin{array}{llllll}0 & 6 & 9 & 12 & 15 & 18\end{array}$ & $\begin{array}{lllll}6 & 9 & 12 & 15 & 18\end{array}$ & days \\
\hline$-----\cdots$ & ----- & Nanog \\
\hline------ & ----1 & Oct -4 \\
\hline 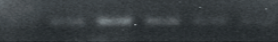 & $---\cdots$ & BMP-2 \\
\hline$-\infty---1$ & $-\infty-\infty$ & BMP-4 \\
\hline 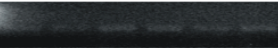 & -- & Bmpr1a \\
\hline----- & ----- & Smad1 \\
\hline------ & ----- & Smad5 \\
\hline----- & $---\infty-$ & GATA-4 \\
\hline 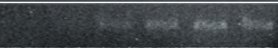 & $-\infty-\infty$ & Nkx2.5 \\
\hline----- & ----- & Cardiac troponin I \\
\hline$--\cdots$ & $---\infty$ & Desmin \\
\hline------ & ----- & GAPDH \\
\hline
\end{tabular}

Figure 4. Induction of BMP signaling molecules and cardiac specific genes in P19 cells exposed to 5-aza, as shown by RT-PCR. RNA was prepared from the 5 -aza-untreated and treated P19 cells on day 0 (undifferentiated P19 cells, lane 1), day 6 (lanes 2 and 7), day 9 (lanes 3 and 8), day 12 (lanes 4 and 9), day 15 (lanes 5 and 10) and day 18 (lanes 6 and 11). RT-PCR was performed with primers for Nanog and Oct-4 as undifferentiated stem cell markers, for BMP-2, BMP-4, Bmpr1a, Smad1 and Smad5 as BMP signaling molecules and for GATA-4, Nkx2.5, cardiac troponin I and desmin as cardiac specific markers, and GAPDH was used as an internal control. expressions of all cardiac markers such as GATA-4, $\mathrm{Nkx2.5}$, cardiac troponin I and desmin were observed in both the 5-aza -untreated and treated P19 cells in a time-dependent manner (Figure 4). However, the expressions of these cardiac markers increased markedly by 5 -aza treatment suggests that 5 -aza treatment specifically enhanced the cardiac differentiation of P19 cells in the confluent monolayer culture (Figure $4)$. The expression of an early cardiac transcription factor, GATA-4, was induced on day 6 in both the 5-aza-untreated and treated P19 cells (Figure 4). The up-regulation of GATA-4 observed at day 9, remained elevated until day 18 in the 5 -aza-treated cells, but not in the control cells (Figure 4). The expression of the undifferentiated stem cell markers, Oct-4 and Nanog, which were the proteins responsible for the maintenance of pluripotency in ES or carcinoma cells (Chambers et al., 2003; Mitsui et al., 2003) was also examined to measure the proportions of the undifferentiated cells after a 5-aza exposure. The levels of undifferentiated stem cell markers, Oct-4 and Nanog strongly expressed in undifferentiated P19 cells, were gradually decreased in both the 5-aza-untreated and treated cells in a time-dependent manner (Figure 4). BMP signaling is known to be essential for P19 cardiac differentiation (Van der Heyden and Defize, 2003). BMP-2 and BMP-4 were highly induced at day 6 or 9 in the 5 -aza-treated cells (Figure 4). The type $1 \mathrm{~A}$ receptor for BMPs, Bmpr1a was also induced by 5-aza at day 6 and 9 (Figure 4) whereas Bmpr1b

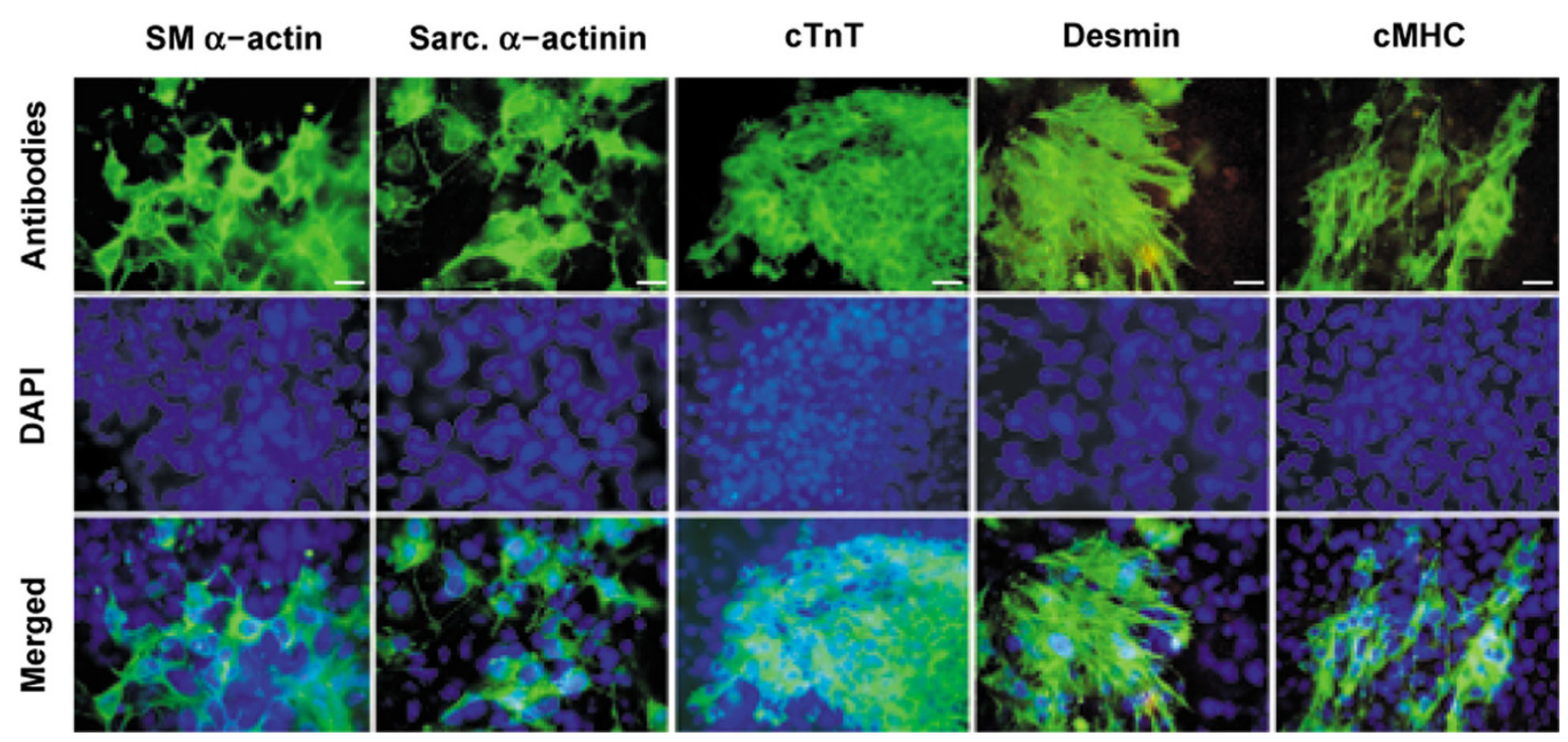

Figure 5. Immunostaining of P19 cell-derived cardiomyocytes after 5 -aza treatment. P19 cells were cultured on $0.1 \%$ gelatin coated coverslips at a density of $1.0 \times 10^{3}$ cells/well in 12-well dishes in the medium containing $1 \mu \mathrm{M}$ of 5 -aza. After 12 days of 5 -aza treatment, the cells were stained with smooth muscle (SM) $\alpha$-actin, sarcomeric (Sarc.) $\alpha$-actinin, cardiac troponin T (cTnT), desmin, cardiac myosin heavy chain (cMHC) as indicated. DAPI was used for staining the nuclei. Scale bar, $30 \mu \mathrm{m}$. 
A
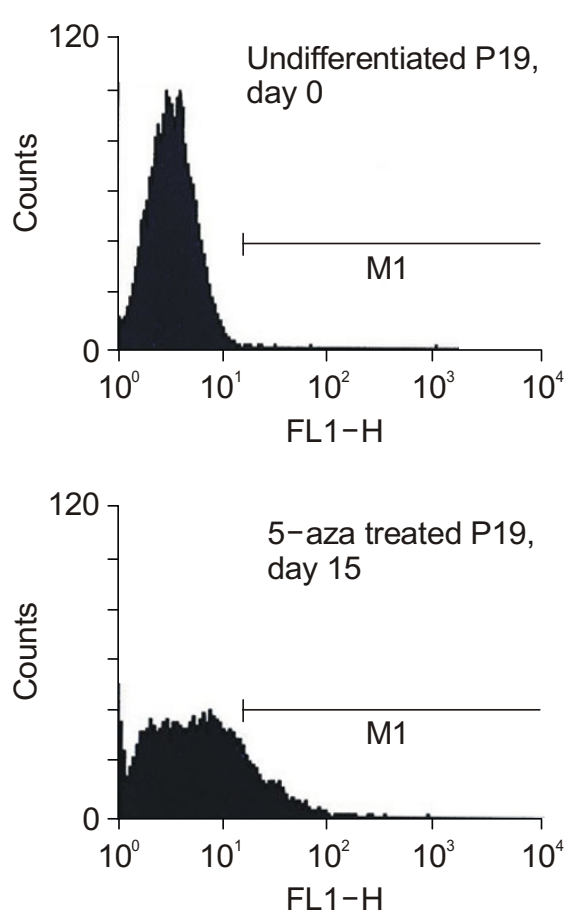

B

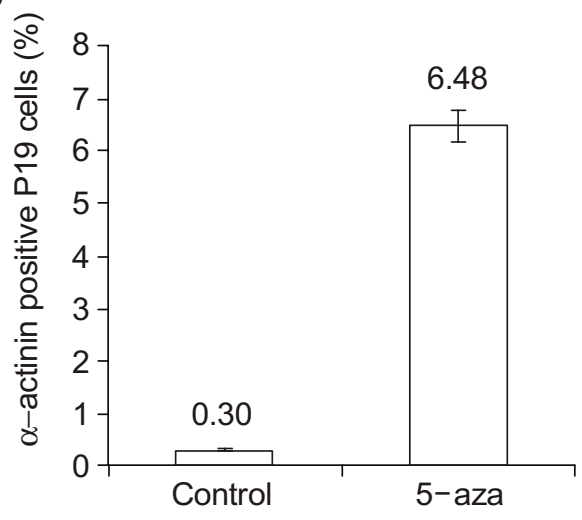

Figure 6. Quantification of P19 cells differentiated into $\alpha$-actinin positive cells after 5-aza treatment. A. 5-aza-treated P19 cells were recovered at 15 days, then stained with an anti-sarcomeric $\alpha$-actinin antibody and analyzed by flow cytometry. The data shown are representative of similar results obtained from three independent experiments. M1 indicates the $\alpha$-actinin positive cells. B. Percentage of P19 cells expressing sarcomeric $\alpha$-actinin at the undifferentiated state after 15 days of treatment with 1 $\mu \mathrm{M}$ of 5 -aza.

expression is not readily detectable in basal or the 5 -aza-untreated cells (data not shown). The expression of Smad1 was moderately stimulated at days 6 and 9 in the 5-aza-treated cells (Figure 4) whereas the induction of Smad5 was not apparent in 5-azatreated cells. In response to 5-aza, the induction of BMP signaling molecules was enhanced at day 6 or 9 , and then the expression of those was reduced to basal level from day 12 (Figure 4), consistent with the early regulatory effects of BMP signaling molecules on cardiac differentiation. None of these observations was seen in the 5-aza-untreated cells. The findings suggest that cardiac differentiation of P19 cells by 5 -aza treatment is at least partially dependent on the un-masking of genes that promote BMP signaling.

To confirm further cardiac differentiation by 5 -aza treatment, we performed immunostaining for the cardiac muscle markers in $\mathrm{P} 19$ cells that were treated with $1 \mu \mathrm{M}$ of 5 -aza for 12 days. 5 -aza induced cardiomyocytes showed positive staining with anticardiac myosin heavy chain, anti-cardiac troponin $\mathrm{T}$, anti-desmin, anti-smooth muscle $\alpha$-actin and antisarcomeric $\alpha$-actinin antibodies, with striations and the staining pattern of the cytoplasmic localization being observed that were characteristic of the sarcomeric organizations of muscle cells (Figure 5).

To quantify the fraction of cardiac differentiated cells by 5 -aza treatment, we analyzed the cardiomyogenic cells by flow cytometry for the muscle specific marker, sarcomeric $\alpha$-actinin. The proportion of sarcomeric $\alpha$-actinin positive cells increased from $0.30 \%$ for the undifferentiated $\mathrm{P} 19$ cells up to $6.48 \%$ on day 15 after the 5 -aza exposure (Figure 6).

\section{Discussion}

Five-aza or its derivatives was reported to induce cardiac differentiation in mesenchymal stem cells derived from bone marrow, and 5-aza or its derivatives can enhance the generation of cardiomyocytes in human ES cells (Makino et al., 1999; Hakuno et al., 2002; Xu et al., 2002; Rangappa et al., 2003). However, the precise mechanisms for the action of 5 -aza on cardiomyocyte differentiation are somewhat unclear, but postulated to be involved in the alteration of gene expression by demethylation or the specific action of 5 -aza that is capable of differentiating human ES cells into cardiomyocytes. In this study, we found that 5-aza, a demethylation agent, induces the differentiation of P19 cells into cardiomyocytes in confluent monolayer culture without both a prior EB formation and DMSO exposure. These results suggest that the demethylation process is an important factor in cardiac differentiation, and it is capable of altering gene expression or regulating cellular differentiation such as BMP signaling cascade. Further studies will be needed to clarify the mechanisms involved in the effect of 5 -aza or its derivatives on cardiac differ- 
entiation.

It is known that the efficient differentiation of P19 cells depends on the prior formation of non-adhering aggregates, and these cellular aggregates resemble the inner cell mass of an embryo, although the molecular events that occur during aggregation and that are necessary for cardiac differentiation are not fully understood (Van der Heyden and Defize, 2003). Skerjanc et al. (1998) have reported that Nkx2.5 can induce cardiomyogenesis in aggregated P19 cells, but not in monolayer cultures. The addition of soluble BMP4 bypassed the requirement for aggregation and induced cardiomyogenesis in monolayer cultures of P19 cells expressing Nkx2.5 (Jamali et al., 2001), and this demonstrated that cellular aggregation is definitely required to generate the BMP signaling in P19 cells. Our study showed that 5-aza altered the expression of BMP signaling molecules in P19 confluent monolayer culture, suggesting that the induction of BMP signaling cascade can bypass the requirement for $E B$ formation and subsequently regulate $\mathrm{Nkx} 2.5$ activity.

It has been reported that 5 -aza treatment can affect transforming growth factor- $\beta$ (TGF- $\beta$ ) and BMP signaling in animal cell systems. 5 -aza has been shown to alter TGF- $\beta$ and BMP signaling via the up-regulation of Smad1 and 5 in articular chondrocytes (Zuscik et al., 2004). 5-aza has also been shown to enhance the expression of TGF- $\beta$ receptors through the down-regulation of $\mathrm{Sp} 3$, a transcriptional repressor of TGF- $\beta$ receptors type $I$ and $I I$, in MCF-7L breast cells and GEO colon cancer cells (Ammanamanchi and Brattain, 2001). The exposure of 5-aza induced cardiac differentiation of heart-derived progenitor cells, which depends, in part, on Bmpr1a, a type IA receptor for BMP (Oh et al., 2004). In our experimental conditions, 5-aza treatment caused a potent induction in the expression of BMP-2 and BMP-4 as well as Bmpr1a and Smad1 specific for the BMP pathway, and this may result in cardiomyogenic differentiation in the absence of EB formation. However, the exact action of 5-aza for inducing cardiomyogenic differentiation in animal cells including MSC and P19 cells remains to be determined.

On the other hand, P19CL6 cells that were derived from original P19 cells efficiently differentiated into cardiomyocytes when they were treated with DMSO in the absence of prior EB formation under adherent conditions at very high cell densities (Habara-Ohkubo, 1996). Several studies have also reported that spontaneous differentiation events that occur in the absence of cellular aggregation could be triggered by high cell densities such as those densities encountered in aggregates (Rudnicki and McBurney, 1987; McBurney, 1993; Paquin et al., 2002). In this context, we thought that the differentiation protocol used in this study, such as that caused from an altered over- all gene expression by the action of 5-aza, and also the differentiation that was caused by an increase in local cell densities, induced cardiac differentiation in P19 cells even though EB formation is bypassed, as in the P19CL6 cell experiments.

Undifferentiated P19 cells showed severe cell death when they were exposed to more than $5 \mathrm{uM}$ of 5-aza in the monolayer, and this happened even though 5 -aza is known to be at least 10 -fold less cytotoxic than 5-aza-2'-deoxycytidine in cell cultures (Flatau et al., 1984; Chrisman, 2002). However, the P19 cell aggregates were resistant to even $10 \mu \mathrm{M}$ of 5 -aza treatment (Figures $1 \mathrm{~A}$ and $1 \mathrm{~B}$ ). In bone marrow derived mesenchymal stem cells, 5-aza or a 5aza-derivative in the range from 3 to $12 \mu \mathrm{M}$ have been used to induce cardiac differentiation (Makino et al., 1999; Hakuno et al., 2002; Rangappa et al., 2003). When these results are examined together, they suggest that the cytotoxicity of 5-aza may have greater potency for undifferentiated embryonic stem cells than the 5-aza cytotoxicity noted for adult stem cells such as mesenchymal stem cells or the differentiated cell aggregates.

In this study, we demonstrated that P19 cells can be differentiated into cardiomyocytes by a 5-aza treatment in the P19 confluent monolayer culture in the absence of prior EB formation and without exposure to DMSO, depending at least in part on alteration of BMP signaling cascade. These findings suggest that 5 -aza treatment in confluent monolayer cultures of P19 cells could be used as a new method or it can be used in combination with other systems to enhance cardiac differentiation. Our results will contribute to elucidating the molecular mechanism of cardiac differentiation.

\section{Acknowledgment}

This study was supported by a grant (01-PJ10-PG801EC01-0027) of the Korea Health 21 R\&D Project, Ministry of Health and Welfare, Republic of Korea.

\section{References}

Ammanamanchi S, Brattain MG. 5-azaC treatment enhances expression of transforming growth factor-beta receptors through down-regulation of Sp3. J Biol Chem 2001;276: 32854-9

Bartolucci S, Estenoz M, de Franciscis V, Carpinelli $P$, Colucci GL, Augusti Tocco G, Rossi M. Effect of cytidine analogs on cell growth and differentiation on a human neuroblastoma line. Cell Biophys 1989;15:67-77

Branch S, Francis BM, Chernoff N. Teratogenic effects of the demethylating agent 5-aza-2'-deoxycytidine in the Swiss Webster mouse. Toxicology 1996;112:37-43

Chambers I, Colby D, Robertson M, Nichols J, Lee S, 
Tweedie S, Smith A. Functional expression cloning of Nanog, a pluripotency sustaining factor in embryonic stem cells. Cell 2003;113:643-55

Chrisman JK. 5-Azacytidine and 5-aza-2'-deoxycytidine as inhibitors of DNA methylation: mechanistic studies and their implications for cancer therapy. Oncogene 2002;21:5483-95

Cihak A. Biological effects of 5 -azacytidine in eukaryotes. Oncology 1974;30:405-22

Edwards MKS, McBurney MW. The concentration of retinoic acid determines the differentiated cell types formed by a teratocarcinoma cell line. Dev Biol 1983;98:187-91

Flatau E, Gonzales FA, Michalowsky LA, Jones PA. DNA methylation in 5-aza-2'-deoxycytidine-resistant variants of C3H 10T1/2 C18 cells. Mol Cell Biol 1984;4:2098-102

Habara-Ohkubo A. Differentiation of beating cardiac muscle cells from a derivative of P19 embryonal carcinoma cells. Cell Struct Funct 1996;21:101-10

Hakuno D, Fukuda K, Makino S, Konishi F, Tomita $Y$, Manabe T, Suzuki $Y$, Umezawa A, Ogawa S. Bone marrowderived regenerated cardiomyocytes (CMG Cells) express functional adrenergic and muscarinic receptors. Circulation 2002;105:380-6

Jamali M, Karamboulas C, Rogerson PJ, Skerjanc IS. BMP signaling regulates $\mathrm{Nkx2-5}$ activity during cardiomyogenesis. FEBS Lett 2001;509:126-30

Jones PA, Taylor SM. Cellular differentiation, cytidine analogs and DNA methylation. Cell 1980;20:85-93

Makino S, Fukuda K, Miyoshi S, Konishi F, Kodama H, Pan J, Sano M, Takahashi T, Hori S, Abe H, Hata J, Umezawa A, Ogawa S. Cardiomyocytes can be generated from marrow stromal cells in vitro. J Clin Invest 1999;103:697-705

Martin GR. Teratocarcinomas as a model system for the study of embryogenesis and neoplasia. Cell 1975;5:229-43

McBurney MW, Jones-Villeneuve EMV, Edwards MKS, Anderson PJ. Control of muscle and neuronal differentiation in a cultured embryonal carcinoma cell line. Nature 1982;299: $165-7$

McBurney MW. P19 embryonal carcinoma cells. Int J Dev Biol 1993;37:135-40

Mitsui K, Tokuzawa $\mathrm{Y}$, Itoh $\mathrm{H}$, Segawa K, Murakami M, Takahashi K, Maruyama M, Maeda M, Yamanaka S. The homeoprotein Nanog is required for maintenance of pluripotency in mouse epiblast and ES cells. Cell 2003;113:631-42

Mohandas T, Sparkes RS, Shapiro LJ. Reactivation of inactive human $X$ chromosome: evidence for $X$ inactivation by DNA methylation. Science 1981;211:393-6

Mummery CL, van Achterberg TA, van den Eijnden-van Raaij AJ van Haaster $L$, Willemse $A$, de Laat SW, Piersma $A H$. Visceral-endoderm-like cell lines induce differentiation of murine P19 embryonal carcinoma cells. Differentiation 1991;46:51-60
Oh H, Bradfute SB, Gallardo TD, Nakamura T, Gaussin V, Mishina Y, Pocius J, Michael LH, Behringer RR, Garry DJ, Entman ML, Schneider MD. Cardiac progenitor cells from adult myocardium: homing, differentiation, and fusion after infarction. Proc Natl Acad Sci USA 2003;100:12313-8

Oh $H$, Chi X, Bradfute SB, Mishina $Y$, Pocius J, Michael LH, Behringer RR, Schwartz RJ, Entman ML, Schneider MD. Cardiac muscle plasticity in adult and embryo by heartderived progenitor cells. Ann N Y Acad Sci 2004;1015:182-9

Overbergh L, Valckx D, Waer M, Mathieu C. Quantification of murine cytokine mRNAs using real time quantitative reverse transcriptase PCR. Cytokine 1999;11:305-12

Paquin J, Danalache BA, Jankowski M, McCann SM, Gutkowska J. Oxytocin induces differentiation of P19 embryonic stem cells to cardiomyocytes. Proc Natl Acad Sci USA 2002;99:9550-5

Rangappa S, Fen C, Lee EH, Bongso A, Wei ES. Transformation of adult mesenchymal stem cells isolated from the fatty tissue into cardiomyocytes. Ann Thorac Surg 2003;75: 775-9

Rodriguez ER, Tan CD, Onwuta US, Parrillo JE. Cardiac myocyte differentiation induced by 3, 5, 3'-triiodo-L-thyronine (T3) in P19 teratocarcinoma cells is accompanied by preferential binding of $R G G(T / A) C A$ direct repeats spaced by 4 base pairs in the DNA. Biochem Biophys Res Commun 1994;205:1899-906

Rudnicki MA, McBurney MW. Cell Culture Methods and Induction of Differentiation of Embryonal Carcinoma Cell Lines. In Teratocarcinomas and Embryonic Stem Cells: A Practical Approach (Robertson EJ, ed), 1987, IRL Press, Oxford, Washington DC

Skerjanc IS, Petropoulos H, Ridgeway AG, Wilton S. Myocyte enhancer $2 \mathrm{C}$ and Nkx2-5 up-regulate each other's expression and initiate cardiomyogenesis in P19 cells. J Biol Chem 1998;52:34904-10

Sorm F, Piskala A, Cihak A, Vesely J. 5-Azacytidine, a new, highly effective cancerostatic. Experientia 1964;20:202-3

Takahashi T, Lord B, Schulze PC, Fryer RM, Sarang SS, Gullans SR, Lee RT. Ascorbic acid enhances differentiation of embryonic stem cells into cardiac myocytes. Circulation 2003;107:1912-6

Van der Heyden, MA, Defize LH. Twenty one years of P19 cells: what an embryonal carcinoma cell line taught us about cardiomyocyte differentiation. Cardiovasc Res 2003;58:292302

Xu C, Police S, Rao N, Carpenter MK. Characterization and enrichment of cardiomyocytes derived from human embryonic stem cells. Circ Res 2002;91:501-8

Zuscik MJ, Baden JF, Wu Q, Sheu TJ, Schwarz EM, Drissi $\mathrm{H}$, O'Keefe RJ, Puzas JE, Rosier RN. 5-azacytidine alters TGF-beta and BMP signaling and induces maturation in articular chondrocytes. J Cell Biochem 2004;92:316-31 